\title{
What is the effect of school closures on learning in Canada? A hypothesis informed by international data
}

\section{À quels effets sur l'apprentissage doit-on s'attendre suite aux interruptions scolaires au Canada? Une hypothèse inspirée de données internationales}

\author{
Catherine Haeck $k^{1,2,3}$ (i) $\cdot$ Simon Larose $\mathrm{e}^{1,4}$ \\ Received: 26 March 2021 / Accepted: 19 August 2021 / Published online: 28 January 2022 \\ (c) The Author(s), under exclusive licence to The Canadian Public Health Association 2021
}

\begin{abstract}
Canadian lockdown response to the COVID-19 pandemic has included province-wide school shutdowns and frequent individual school closures to contain outbreaks. A number of scientists and political figures have shared their concerns about the medium- and long-term effects of school closures/shutdowns on student academic achievement, learning loss, and learning gaps. Unfortunately, there are no pan-Canadian studies to date to help define the scope of the problem. In this commentary, we report the results of a number of longitudinal research studies conducted in the Netherlands, Belgium, England, and the United States. Using these studies as a basis for comparison, we extrapolated a "Canadian" hypothesis on the unintended academic consequences of school closures, keeping in mind the unique nature of each province. We continue with recommendations on the types of research required to validate this hypothesis, and conclude with implications on public health and education should learning loss and gaps prove true.
\end{abstract}

\section{Résumé}

La pandémie de Covid-19 a entrainé la fermeture temporaire de plusieurs écoles au Canada ainsi que des interruptions scolaires fréquentes en raison d'éclosions. Plusieurs scientifiques et politiciens ont partagé leurs inquiétudes sur la place publique quant aux effets à moyen et long terme de cette situation sur la réussite des élèves. Malheureusement, aucune étude pancanadienne ne permet à ce jour de bien circonscrire l'ampleur du problème. Dans ce commentaire, nous rapportons le fruit des quelques recherches longitudinales réalisées ailleurs dans le monde sur cette question (c.-à-d. Pays Bas, Belgique, Angleterre et États-Unis). En utilisant ces recherches comme base de comparaison, nous nous avançons sur ce que serait l'hypothèse « canadienne» tout en gardant en tête les réalités très singulières de chaque province. Nous concluons le commentaire par des recommandations sur le type de recherche à mettre en œuvre pour valider cette hypothèse et sur les implications pour l'éducation et la santé publique si elle s'avérait confirmée.

Keywords COVID-19 $\cdot$ Lockdown $\cdot$ School closures $\cdot$ Learning loss $\cdot$ Academic achievement $\cdot$ Education

Mots-clés COVID-19 · interruptions · fermeture d'écoles · perte d'apprentissage · réussite scolaire · éducation

Lockdown response to the COVID-19 pandemic in school settings has affected the quality and quantity of academic

Simon Larose

simon.larose@fse.ulaval.ca

Extended author information available on the last page of the article schoolwork and extracurricular activities across Canada and has indirectly fragilized the pedagogical process. These measures included province-wide school shutdowns starting mid-March 2020, with online teaching added as an emergency measure, followed by class bubbles, and frequent individual school closures to contain outbreaks later in the 
fall of 2020. Many Canadian experts (e.g., Nemer et al., 2020) expect to see significant learning loss, particularly among the most vulnerable students, such as children/ teens from disadvantaged or low-income families or with special needs, but no pan-Canadian high-quality education data source can help validate these expectations. However, previous research (prior to the pandemic) showed that the gap between high- and low-performing students in reading, mathematics, and science was significant and relatively comparable between provinces (Haeck \& Lefebvre, 2021). Although Canada ranks among the most egalitarian of nations, low-performing students at age 15 do not possess the level of competence required for post-secondary studies, as attested by seven Programme for International Student Assessment (PISA) surveys, 2000-2018.

Haeck and Lefebvre (2020) expect a 30\% increase in performance gap in Canada as a result of pandemic-induced school shutdowns. This view is shared by US projections (Kuhfeld et al., 2020a). Further and notably, Hanushek and Woessmann (2020) estimated that the present value of lost gross domestic product (GDP) for Canada over 80 years due to pandemic-induced learning loss would equal \$1272 billion USD, or the equivalent of one year's GDP. These studies are based on predictions, since there are as yet no population-based longitudinal or cross-sectional studies in Canada with standardized data to compare actual learning in pre- and post-COVID-19 cohorts. Furthermore, since provinces cancelled elementary and high school government exams, making a definitive assessment of the impact of the pandemic's short-term impacts will be nearly impossible. We therefore have to rely on research initiatives from other countries such as the Netherlands, Belgium, and the United States for comparison.

In the Netherlands, Engzell et al. (2021) studied the impact of school closures on academic performance in elementary school. Results from the Netherlands must be considered conservative, as in-person schooling was only briefly suspended ( 8 weeks) in the pandemic first wave. Further, broadband access is widely available and technological preparedness among teaching staff was relatively high. Regular semi-annual national testing of some 350,000 students (math, spelling, reading) took place a few weeks before and after the lockdown. Results revealed an overall learning loss of about 0.08 standard deviations, which represents about one quarter of a year of learning. Losses were $55 \%$ higher in students from less-educated homes, as compared with others.

In Belgium, schools closed for 9 weeks. Online learning started unevenly 4 weeks after lockdown. In a longitudinal study (2015-2020) of standardized year-end testing of several thousand students in their last year of Flemish elementary schools, Maldonado and De Witte (2020) found a grade point average (GPA) loss of 0.25 standard deviations in 2020, as compared with previous cohorts: $0.18-0.25$ standard deviations for math, 0.29 for Dutch, 0.19-0.30 for French, and 0.07-0.16 for science. Learning loss was greater in disadvantaged neighbourhoods and in schools where maternal education levels were lower.

In the USA, to our knowledge, there are no studies to date with representative population samples and school testing to measure pandemic-related learning loss due to school closures. However, Kuhfeld et al. (2020a) created a longitudinal model using data from 5 million students in Grades 3-8 in 2017-2019. They compared typical learning trajectories with three scenarios: partial absenteeism (assuming students received half of their normal instruction in spring 2020), COVID slide (spring 2020 school closures likened to a typical summer break), and full absenteeism. Results suggested learning losses for the 2020-2021 cohort of $32-37 \%$ for reading and $50-63 \%$ for mathematics. More recent preliminary data on actual learning losses (Kuhfeld et al., 2020b) confirmed these predictions for mathematics, less so for reading.

In the United Kingdom, a study by Andrew et al. (2020) highlighted a possible reason for the association between learning gaps and socio-economic status (SES). At the beginning of the pandemic, learning time and access to technology and educational resources were greater in wellto-do families than in poorer ones. This phenomenon has been observed in the USA (Chetty et al., 2020) and Canada (Frenette et al., 2020). However, literature on teacher strikes (e.g., Jaume \& Willen, 2019; Johnson, 2011) and summer vacations (e.g., Davies \& Aurini, 2013) also demonstrated a widening gap related to SES. In such instances, there was no question of access to technology.

The portrait painted so far by the few international studies to date on the effect of pandemic-induced school closures is not reassuring for Canada. Based on UNESCO data, Canada is among the countries reporting the most school closures during lockdown, with all provinces doing so at some point (Fig. 1). As of March 29, 2021, according to UNESCO data, schools in Canada had closed for 40 weeks: 13 full-time and 27 part-time for high school students. Considering that online teaching was deployed unevenly, that a number of education specialists consider online learning as a suboptimal teaching mode (Marshall et al., 2020) and that countless schools closed due to outbreaks during the 2020-2021 school year, it is imperative to quickly assess the situation in Canada. Based on the evidence from the few studies conducted elsewhere, we hypothesize that academic setbacks will be significant in Canada. Particularly affected will be vulnerable students: children/teens with special needs, special challenges, poor marks in school, or from disadvantaged families. 
Fig. 1 Full-time and parttime school closures in weeks by country, using data from UNESCO (https://en.unesco. org/covid19/educationrespon se\#durationschoolclosures). Source: Calculations by the authors. Data last updated on March 29, 2021

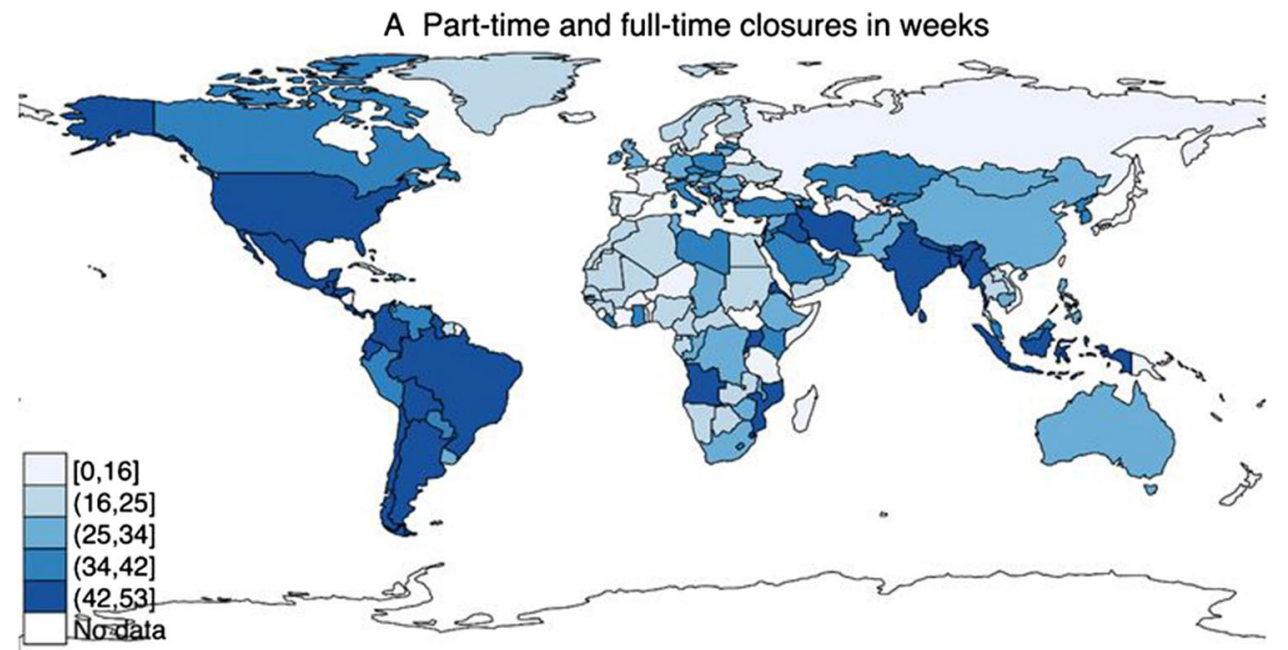

B Full-time closures in weeks

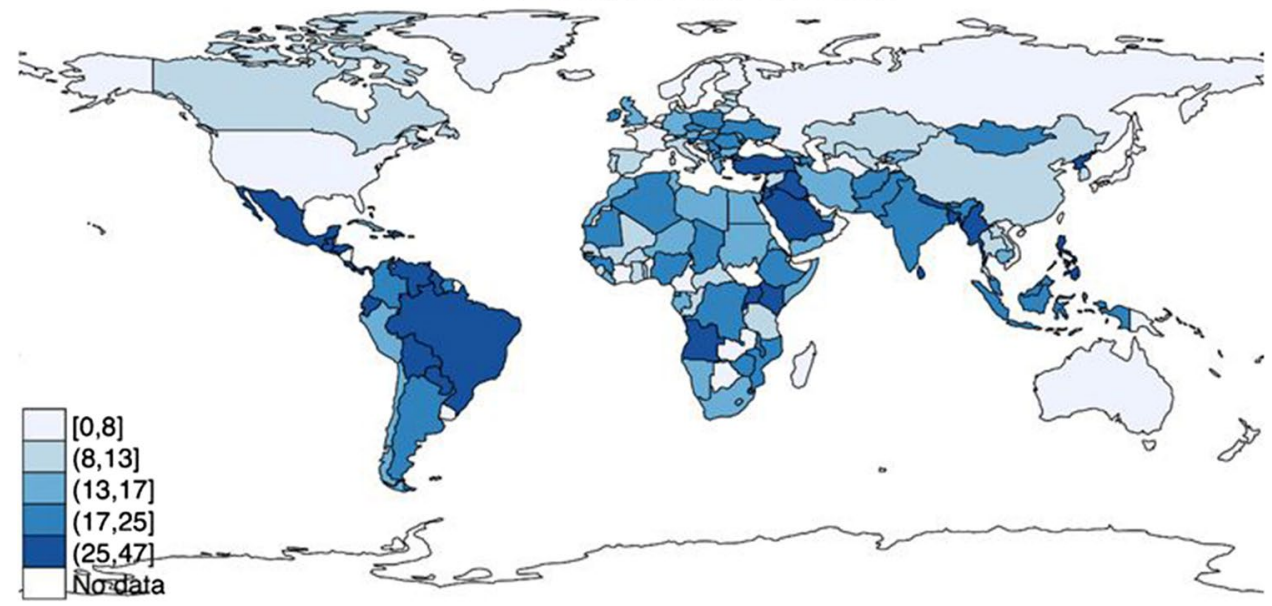

The consequences of optimal or suboptimal school performance on quality of life in adulthood are well documented. An increase in school performance of 0.2 standard deviation was associated with an increase in total lifetime income of $2.6 \%$ on average, as well as a greater likelihood of participation in the labour market (Chetty et al., 2014). Further, learning losses from teacher strikes have been associated with lower income and a lower probability of employment in adulthood, and poorer school attainment in the next generation (Jaume \& Willen, 2019). Because of potential intergenerational effects of school closures and because educational losses are often greater felt in disadvantaged neighbourhoods and low achievers, pandemicinduced school shutdowns might actually increase social inequalities in Canada in the medium and long term as well.

This brief status report highlights the necessity of conducting a systematic study of the effects of school closures on learning and success in young people across Canada. With no standardized tests/government exams in 2020 and 2021, and no cross-Canada surveys documented so far, it will be difficult to reliably measure the short-term effects of the pandemic. However, other studies with representative sample data signal the possibility of medium- to long-term effects of school interruptions. To be able to quickly correct the situation in those more deeply affected by the pandemic, it is essential to conduct multi-cohort longitudinal studies to ideally include pre-, peri-, and post-pandemic standardized measures of basic skills (language, mathematics, science) as well as data on school interruptions. Public health, provincial ministries of education and social services, and local school boards must clearly report absenteeism, providing researchers with reliable data per school, including number of days closed, number of COVID cases, class bubbles, and temporary closures for COVID outbreaks. Parents need to be surveyed to measure time spent on learning at home (Andrew et al., 2020) and their perceptions of the accessibility and efficiency of online education. Teachers need to be surveyed to provide researchers with data on mental health and selfperceived mastery of technological tools; these factors may act as mediators or moderators of the effect of school interruptions on student learning and academic success. 
Should findings in Canada confirm our hypothesis, several avenues of intervention should be considered to limit the effects of school closures on learning and academic success. Governments could subsidize the hiring of tutors with expertise in particular subject areas and knowledge of the particular school culture and teacher expectations of the students they tutor. A systematic review on tutoring (Nickow et al., 2020) found that tutoring by teachers and professional educators during school hours resulted in better marks than tutoring by peers and adult volunteers. If tutors are not professional educators, they should at a minimum receive initial training and be supervised by experienced teachers (Larose \& Tarabulsy, 2014). The government could also work with community organizations to ensure that summer camps include course catch-up material within their schedules, although effectiveness remains to be determined. Informal background education does have the potential to consolidate and enrich subject matter and prepare students for the following school year. Last, it would be useful to coordinate course work between teachers of the preceding and succeeding years once school starts in the fall. Although barely studied, coordination between elementary and high school teachers is uncommon, and between high school and post-secondary quasi-nonexistent. Considering how difficult these transitions can be, especially for at-risk students (Larose et al., 2018), it is imperative that teachers identify exactly what was learned, how much subject material remains to be consolidated, and what aspects to prioritize in the coming year.

\section{Conclusion}

COVID-19 has impacted more than physical health. In Canada as elsewhere, it is important to measure the unintended consequences of pandemic lockdown on education losses/ gaps and academic success, so as to quickly mitigate any potential negative effects.

\section{À quels effets sur l'apprentissage doit-on s' attendre suite aux interruptions scolaires au Canada? Une hypothèse inspirée de données internationales}

La pandémie de Covid-19 et les mesures de confinement des populations scolaires adoptées par les gouvernements provinciaux canadiens ont affecté la quantité et la qualité des enseignements et services scolaires offerts par les écoles et, indirectement, fragilisé les processus d'enseignement. Ces mesures incluent, entre autres, la fermeture complète des écoles dès la mi-mars 2020 à travers le pays, l'enseignement à distance mis en place en mode d'urgence, suivi par la création de bulles-classes et les fermetures temporaires de classes pour contrôler les éclosions à l'automne 2020. Plusieurs experts canadiens anticipent que le contexte actuel entraînera d'importants retards scolaires, notamment chez les populations d'enfants plus vulnérables (p.ex., milieux défavorisés, élèves handicapés ou en difficulté d'adaptation ou d'apprentissage) (p.ex. Nemer et al., 2020). À ce jour, aucune source de données canadiennes ne permet cependant de valider cette hypothèse, en partie parce que les épreuves standardisées ont été annulées durant la pandémie. Par contre, nous savons que les écarts d'apprentissage entre élèves canadiens performants et moins performants en lecture, mathématiques et sciences sont importants et relativement comparables d'une province à l'autre (Haeck $\&$ Lefebvre, 2021). Bien que le Canada soit parmi les pays les plus égalitaires, il n'en demeure pas moins que les élèves les moins performants ont un niveau de compétence à l'âge de 15 ans insuffisant pour poursuivre des études post-secondaires. Ces constats proviennent des sept cycles d'enquêtes PISA (Programme for International Student Assessment) menés entre les années 2000 et 2018. Ainsi, déjà avant que ne frappe la pandémie, un nombre important de jeunes canadiens avaient des retards d'apprentissage importants.

Haeck et Lefebvre (Haeck \& Lefebvre, 2020) anticipent un accroissement des inégalités de réussite de près de $30 \%$ au Canada à la suite des fermetures d'écoles entraînées par la pandémie, point de vue qui est fortement partagé par les projections américaines (Kuhfeld et al., 2020a). Hanushek et Woessmann (Hanushek \& Woessmann, 2020) estiment quant à eux que les retards d'apprentissage auront un impact négatif sur le produit intérieur brut (PIB) du Canada d'au moins 1272 milliards USD, soit l'équivalent de près d'un an de PIB sur 80 ans. Ces études se basent sur des prévisions, car à l'heure actuelle, il n'existe au Canada aucune étude populationnelle, longitudinale et/ou transversale, qui inclut des données standardisées sur les apprentissages des élèves avant et depuis la pandémie, ou encore qui permet de comparer les apprentissages des élèves de la Covid-19 à ceux de cohortes antérieures. De plus, comme une majorité de provinces ont annulé leurs épreuves ministérielles à l'enseignement primaire et secondaire, il s'avère difficile de porter un jugement définitif sur l'évolution des apprentissages des élèves. Ainsi, pour anticiper les impacts des fermetures sur les apprentissages des élèves, nous devons nous tourner vers des études faites à l'extérieur du Canada. Certaines initiatives de recherche menées dans des pays comme les Pays-Bas, la Belgique et les États-Unis offrent des bases de comparaison intéressantes. 
Fig. 1 Fermetures partielles et complètes par semaine en fonction des pays selon des données de l'UNESCO[traduction libre] (https://en.unesco.org/covid 19/educationresponse\#durationsc hoolclosures). Source : Calculs faits par les auteurs. Dernière mise à jour des données le 29 mars 2021

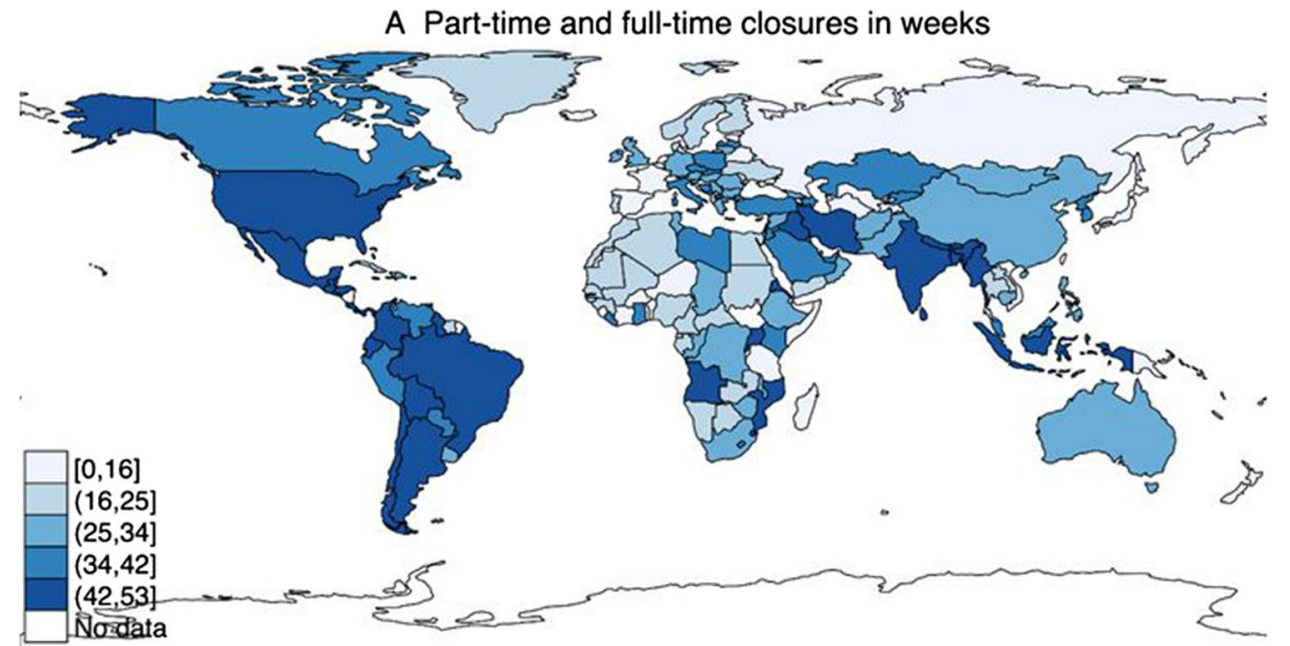

B Full-time closures in weeks

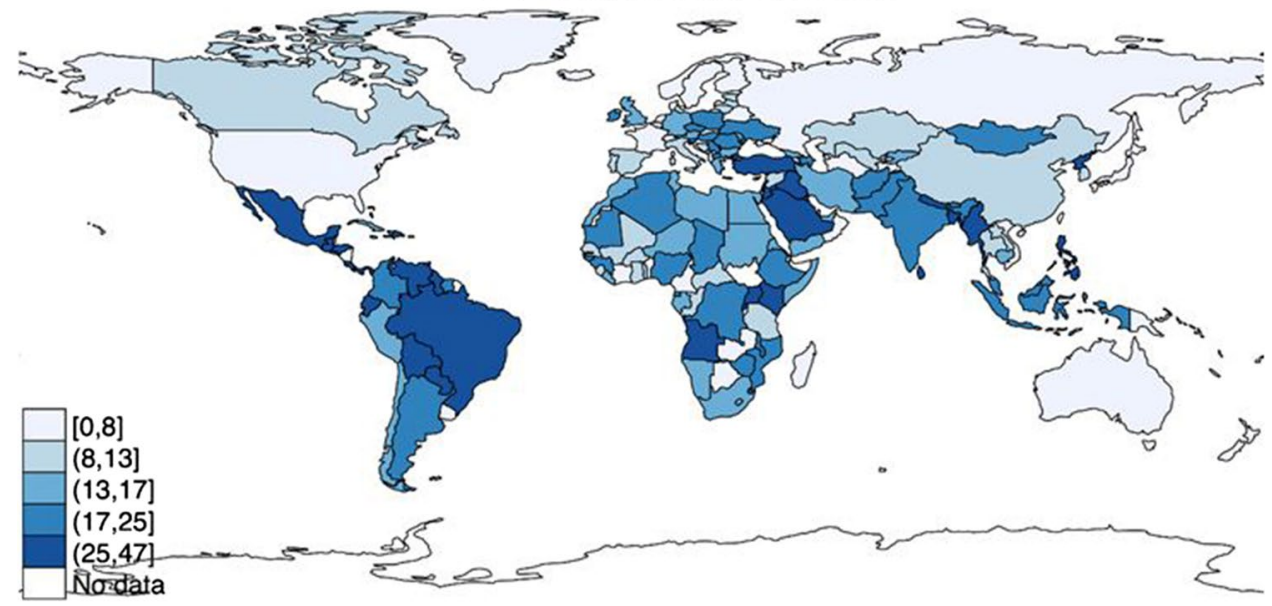

Aux Pays-Bas, Engzell et al. (2021) ont étudié l'impact des fermetures d'écoles sur les résultats scolaires des élèves du primaire. Les Pays-Bas constituent un cas conservateur en raison de la brièveté des fermetures d'écoles lors de la première vague de la pandémie ( 8 semaines) et d'un degré de préparation techno-pédagogique relativement élevé du personnel scolaire. L'étude a impliqué une analyse des résultats de près de 350000 élèves à des évaluations standardisées en mathématique, orthographe et compréhension de lecture. Ces évaluations ont été administrées quelques semaines avant (mars 2020) et après (mai 2020) la fermeture des écoles au moment de la première vague de la pandémie. Les élèves ont montré des pertes moyennes d'apprentissage 0,08 écart-type ce qui représente près d'un quart des acquis fait dans une année normale. Ces pertes ont été $55 \%$ plus importantes chez les enfants dont les parents avaient un faible niveau d'éducation.

La Belgique offre aussi une base de comparaison intéressante. Les écoles ont fermé pendant 9 semaines et l'enseignement en ligne n'a été dispensé que 4 semaines après la fermeture mais de façon très inégale d'une école à l'autre. Dans une étude longitudinale impliquant des évaluations printanières annuelles des compétences disciplinaires menées entre 2015 et 2020 auprès de milliers d'élèves de la communauté flamande, Maldonado et De Witte (Maldonado \& De Witte, 2020) ont trouvé que les élèves de la cohorte 2020 ont vu leurs résultats scolaires diminuer de 0,18 à 0,25 écart-type en mathématiques, de 0,29 écart-type en flamand, et de 0,19 à 0,30 écart-type en français. En sciences, les pertes d'apprentissage ont été moins importantes, avoisinant entre 0,07 et 0,16 écart-type. Finalement, la moyenne globale des élèves a elle aussi diminué de 0,25 écart-type. Enfin les pertes d'apprentissage étaient plus importantes dans les écoles situées dans des quartiers plus défavorisés et dans les écoles où les mères ont un niveau d'éducation moyen plus faible.

À notre connaissance, aucune étude basée sur des données représentatives de tous les élèves et des tests passés à l'école n'a encore mesuré l'effet des interruptions scolaires liées à la Covid-19 sur les apprentissages des élèves américains. Cependant, Kuhfeld et al. (2020a) ont modélisé ces effets à partir des données longitudinales d'un 
échantillon de plus de 5 millions d'élèves de la $3^{\mathrm{e}}$ à la $8^{\mathrm{e}}$ année suivis entre 2017 et 2019. Ils ont établi des trajectoires d'apprentissages normatives sur la base de ces données et comparé ces trajectoires à trois scénarios : l'absentéisme partiel pendant la pandémie (chaque journée de fermeture équivaut à $50 \%$ des gains d'apprentissage habituel), le «Covid slide» (chaque journée de fermeture équivaut au gain d'apprentissage documenté par l'effet empirique des vacances d'été pré-Covid) et l'absentéisme complet (chaque journée de fermeture équivaut au gain d'apprentissage documenté par l'effet empirique de l'absentéisme pré-Covid). Les résultats de leur modélisation suggèrent que les cohortes d'élèves 2020-2021 subiront des pertes dans leurs apprentissages variant entre $32 \%$ et $37 \%$ en lecture et entre $50 \%$ et $63 \%$ en mathématique. Des données préliminaires plus récentes sur les apprentissages réels des élèves à l'automne 2020 confirment ces prédictions en mathématique mais atténuent celles avancées par les chercheurs en lecture (Kuhfeld et al., 2020b).

Enfin, une étude britannique (Andrew et al., 2020) a mis en lumière l'une des raisons pouvant expliquer l'accroissement des écarts d'apprentissage selon le statut socioéconomique durant la pandémie. Elle montre qu'en début de pandémie, le temps d'apprentissage, et l'accès aux technologies et aux ressources éducatives ont été plus élevés chez les élèves de familles ayant un revenu supérieur que chez ceux de familles plus pauvres. Ce phénomène s'observe aussi aux États-Unis (Chetty et al., 2020) et au Canada (Frenette et al., 2020). Cependant, la littérature sur les écarts d'apprentissage découlant des grèves enseignantes (p.ex. Jaume \& Willen, 2019; Johnson, 2011) ou des interruptions scolaires durant l'été (p.ex. Davies \& Aurini, 2013) concluent aussi à un accroissement des écarts selon le statut socioéonomique. Bien entendu, dans ces cas, l'accès à la technologie n'était pas en cause.

Le portrait des quelques études internationales conduites à ce jour sur l'effet des interruptions scolaires en temps de pandémie n'a rien de rassurant pour les élèves canadiens. À partir de données de l'UNESCO sur les fermetures d'écoles à travers le monde, la figure 1 montre clairement que le Canada fait partie des pays qui ont insisté le plus sur cette mesure de confinement en la généralisant à l'ensemble des provinces. En date du 29 mars 2021, selon les données de l'UNESCO, les fermetures atteignaient 40 semaines au Canada, incluant 13 semaines à temps plein et 27 semaines à temps partiel pour les élèves du secondaire. Considérant que l'enseignement à distance fut déployé de manière inégale, que plusieurs spécialistes de l'éducation jugent ce contexte peu favorable aux apprentissages des élèves (Marshall et al., 2020) et qu'un nombre non négligeable d'écoles canadiennes a dû refermer ses portes en raison d'éclosions durant l'année scolaire 2020-2021, il est impératif de documenter rapidement la situation canadienne.
À la lumière de ce portrait et des quelques recherches menées ailleurs dans le monde, nous soulevons l'hypothèse que les retards scolaires seront importants chez les élèves canadiens, notamment dans les milieux défavorisés et dans les populations académiquement plus vulnérables (p.ex., étudiants handicapés ou ayant des besoins spéciaux et étudiants peu performants).

Les conséquences de la réussite scolaire et des retards d'apprentissage sur la qualité de vie à l'âge adulte sont bien connues et imposent un examen rapide des trajectoires d'apprentissage des élèves canadiens en contexte de pandémie. Nous savons qu'une augmentation des résultats scolaires de 0,2 écart-type est associée à une augmentation des revenus totaux sur une vie de $2,6 \%$ en moyenne ainsi qu'à la probabilité d'une plus grande participation au marché du travail (Chetty et al., 2014). L'étude de Jaume et Willen (Jaume \& Willen, 2019) suggère que les pertes d'apprentissage attribuables aux interruptions scolaires dues à des grèves des enseignants sont associées à une probabilité d'emploi et des revenus plus faibles à l'âge adulte, et à l'insuccès scolaire de la génération suivante. Il existerait en effet une potentielle transmission intergénérationnelle des effets des interruptions scolaires. Enfin, il faut retenir que les effets des interruptions scolaires sont souvent plus grands dans les milieux défavorisés et auprès des élèves moins performants, ce qui laisse croire que la pandémie pourrait accroître certaines inégalités sociales au Canada.

Ce bref état de situation impose une étude systématique pancanadienne des effets des interruptions scolaires sur les apprentissages et la réussite des jeunes. Les épreuves standardisées de 2020 et 2021 n'ont pas eu lieu et aucune enquête pancanadienne n'a été menée en 2020-2021. Ainsi, il sera difficile de mesurer les effets à court terme de la pandémie sur les enfants et les jeunes de manière fiable. Par contre, étant donné les constats d'autres études ayant eu accès à des données représentatives, il est important de vérifier les effets à moyen terme afin de pouvoir rapidement corriger la situation dans les milieux davantage touchés par la pandémie. Il est donc impératif de conduire des études longitudinales multi-cohortes qui, idéalement, incluraient des mesures pré, pandémie et post-pandémie. Il est aussi nécessaire que ces études incluent des évaluations standardisées des apprentissages dans les disciplines fondamentales (c.-à-d. langue première, mathématique, sciences) avant et après la pandémie, ainsi que les données sur la configuration des interruptions scolaires dans les écoles canadiennes. Il est aussi essentiel que la santé publique, les ministères de l'éducation et les centres de services et commissions scolaires dressent des portraits clairs de l'absentéisme scolaire en fournissant des données fiables aux chercheurs sur le nombre de journées de fermeture et cas de Covid-19 par école et le nombre d'arrêts temporaires de bulles-classes 
en fonction d'éclosions. Il serait aussi important que les parents soient questionnés sur le temps d'apprentissage des enfants à la maison (Andrew et al., 2020) et sur leurs perceptions de l'accessibilité et de l'efficacité de l'enseignement en ligne. Enfin, des données sur la santé psychologique des enseignants et leurs perceptions de la maîtrise des outils technologiques seraient importantes à colliger puisque celles-ci peuvent médier ou modérer les effets des interruptions scolaires.

Advenant que l'hypothèse canadienne soit confirmée, différentes pistes d'intervention pourraient être envisagées afin de limiter les effets des interruptions scolaires. Les gouvernements devraient financer l'embauche de tuteurs qui ont des expertises disciplinaires, et qui connaissent la culture et les attentes de réussite des enseignants incluant des élèves de l'école fréquentée par les tutorés. Dans une recension exhaustive des écrits sur le tutorat, Nickow et al. (2020) concluent que le tutorat pratiqué par des enseignants et des professionnels de l'éducation durant les heures d'école donne de meilleurs résultats que le tutorat par des pairs ou des adultes bénévoles. Si les tuteurs ne sont pas des professionnels, il devient alors important qu' ils reçoivent une formation pédagogique initiale et qu'ils soient supervisés par des enseignants d'expérience (Larose \& Tarabulsy, 2014). Les gouvernements pourraient également outiller les organisations communautaires responsables des camps d'été afin de les amener à inclure dans leur programmation estivale des activités de rattrapage disciplinaire, bien que l'efficacité de cette approche reste à démontrer. À travers l'éducation informelle, il demeure possible de consolider et enrichir des notions disciplinaires et, ainsi, préparer les élèves à la prochaine année scolaire. Enfin, il pourrait être utile de faciliter la concertation disciplinaire et pédagogique inter-ordres entre enseignants. Bien que les effets de la concertation sur les apprentissages soient peu étudiés, on sait qu'elle est peu fréquente entre le primaire et le secondaire et presque totalement absente entre le secondaire et le post-secondaire alors même que les transitions scolaires fragilisent parfois les cheminements des élèves plus à risque (Larose et al., 2018).

\section{Conclusion}

Il n'est pas seulement sur la santé physique que la Covid-19 a eu des impacts. Au Canada ainsi que d'autre part, il est impératif d'identifier ce qui a été appris pendant la pandémie, ce qui doit être consolidé et ce qui est prioritaire pour la suite de la formation afin de rapidement endiguer les effets négatifs ayant pu découler des interruptions scolaires et de la pandémie plus généralement.

Acknowledgements/Remerciements The authors thank Danielle Buch, Medical Writer, Research, for the translation and revision of the manuscript. / Les auteurs remercient Danielle Buch pour la traduction et révision du manuscrit.

\section{Declarations}

Conflict of interest The authors declare no competing interests.

\section{References/Références}

Andrew, A., Cattan, S., Costa Dias, M., Farquharson, C., Kraftman, L., Krutikova, S., Phimister, A., \& Sevilla, A. (2020) Inequalities in children's experiences of home learning during the COVID-19 lockdown in England. Institute for Fiscal Studies.

Chetty, R., Friedman, J.N., Hendren, N., \& Stepner, M. (2020) How did COVID-19 and stabilization policies affect spending and employment? A new real-time economics tracker based on private sector data. NBER. Working Paper 2743.

Chetty, R., Friedman, J. N., \& Rockoff, J. E. (2014). Measuring the impacts of teachers II: Teacher value-added and student outcomes in adulthood. American Economic Review, 104(9), 2633-2679. https://doi.org/10.1257/aer.104.9.2633

Davies, S., \& Aurini, J. (2013). Summer learning inequality in Ontario. Canadian Public Policy/analyse De Politiques, 39(2), 287-307. https://doi.org/10.3138/cpp.39.2.287

Engzell, P., Frey, A., \& Verhagen, M D. (2021) Learning inequality during the Covid-19 pandemic. Proceedings of the National Academy of Sciences.

Frenette, M., Frank, K., \& Deng, Z. (2020) COVID-19 pandemic: School closures and the online preparedness of children. Statistics Canada, Social Analysis and Modeling Division. Catalogue no. 45280001.

Haeck, C., \& Lefebvre, P. (2020). Pandemic school closures may increase inequality in test scores. Canadian Public Policy, 46(S1), S82-S87. https://doi.org/10.3138/cpp.2020-055

Haeck, C., \& Lefebvre, P. (2021). Trends in cognitive skills inequalities by socioeconomic status across Canada. Canadian Public Policy, 47(1), 88-116. https://doi.org/10.3138/cpp. 2019-039

Hanushek, E.A., \& Woessmann, L. (2020) The economic impacts of learning losses, OECD.

Jaume, D., \& Willen, A. (2019). The long-run effects of teacher strikes: Evidence from Argentina. Journal of Labor Economics, 37(4), 1097-1139.

Johnson, D. (2011). Do strikes and work-to-rule campaigns change elementary school assessment results? CanadianPublic Policy/ analyse De Politiques, 37(4), 479-494. https://doi.org/10.3138/ cpp.37.4.479

Kuhfeld, M., Soland, J., Tarasawa, B., Johnson, A., Ruzek, E., \& Liu, J. (2020a). Projecting the potential impact of Covid-19 school closures on academic achievement. Educational Researcher, 49(8), 549-565.

Kuhfeld, M., Tarasawa, B., Johnson, A., Ruzek, E., \& Lewis, K. (2020b). Learning during COVID-19: Initial findings on students' reading and math achievement and growth. NWEA.

Larose, S., Duchesne, S., Litalien, D., Denault, A.-S., \& Boivin, M. (2018). Adjustment trajectories during the college transition: Types, personal and family antecedents, and academic outcomes. Research in Higher Education, 60, 684-710. https:// doi.org/10.1007/s11162-018-9538-7 
Larose, S. \& Tarabulsy, G. (2014). Academically at-risk students (p. 303-314). In David L. DuBois and Michael J. Karcher (Eds). Handbook of youth mentoring ( $2^{\text {nd }}$ edition). Los Angeles: Sage Publications.

Marshall, D. T., Shannon, D. M., \& Love, S. M. (2020). How teachers experienced the COVID-19 transition to remote instruction. Phi Delta Kappan, 102(3), 46-50. https://doi.org/ 10.1177/0031721720970702

Maldonado, J. \& De Witte, K. (2020). The effect of school closures on standardised student test outcomes. British Educational Research Journal. https://doi.org/10.1002/berj.3754

Nemer, M., Benseler, S., Chambers, C., Cousineau, D., Grunebaum, E., Hall, J., Kubes, P., Langley, J., Laxer, R. M., Plint, A., Polychronakos, C., Quach, C., Sharma, S., \& Bourque-Viens, A. (2020) La COVID-19 et les enfants, Rapport d'un groupe de travail spécial dirigé par la conseillère scientifique en chef du Canada https://www.ic.gc.ca/eic/site/063.nsf/fra/h_98101.html

Nickow, A., Oreopoulos, P., \& Quan, V. (2020). The impressive effects of tutoring on preK-12 learning: A systematic review and meta-analysis of the experimental evidence. NBER Working Paper, No. 27476.

World Health Organization (2021). Data on school closures. https://ourwo rldindata.org/grapher/school-closures-covid?time=2021-03-19

Publisher's note Springer Nature remains neutral with regard to jurisdictional claims in published maps and institutional affiliations.

\section{Authors and Affiliations}

\section{Catherine Haeck $^{1,2,3}$ (1) $\cdot$ Simon Larose ${ }^{1,4}$ (i)}

1 Observatoire pour l'éducation et la santé des enfants, Montréal, Québec, Canada

2 Département de sciences économiques, Université du Québec à Montréal, Montréal, Québec, Canada Canada

4 Département d'études sur l'enseignement et l'apprentissage, Université Laval, Québec, Québec, Canada 